\title{
Traveling wave oscillations in the optical region: a theoretical examination
}

\author{
Amnon Yariv and Desmond R. Armstrong \\ California Institute of Technology, Pasadena, California 91109 \\ (Received 9 October 1972) \\ The interaction of an electron beam with the optical field of a periodically perturbed dielectric \\ thin-film waveguide is considered. When the electron velocity is nearly equal to that of one of the \\ spatial harmonics of the field, a coherent exchange of energy can take place. This interaction, which \\ can lead in principle to a voltage-tunable optical oscillation, is examined in the light of presently \\ available thin-film techniques. An estimate of the oscillation threshold is made.
}

In this paper we consider the possibility of generating optical radiation by means of the interaction between an electron beam moving in vacuum in close proximity to a corrugated thin-film optical waveguide. The corrugation causes the confined optical field to propagate in a form of an infinite number of spatial harmonics whose propagation constants differ by integral multiples of $2 \pi / L$, where $L$ is the corrugation period. ${ }^{1}$ When velocity synchronism is obtained between one of these harmonics and the electron beam, a coherent exchange of energy between the two can take place. Current modulation on the beam excites a wave in the guide which in turn modulates the beam even further. This can lead to optical oscillation at a frequency which can be tuned by varying the electron velocity in a manner similar to that of microwave traveling wave oscillators. ${ }^{2}$

A possible embodiment of such a device is illustrated in Fig. 1. Regions 1 and 3 are dielectrics with indices of refraction $n_{1}$ and $n_{3}$. For a confined mode optical propagation it is necessary that $n_{1}$ be greater than $n_{3}$. The electric field of a TM mode is given by a sum of spatial harmonics:

$$
E_{z}(x, z, t)=\sum_{m=-\infty}^{\infty} E_{m}(x) \exp \left[i\left(\omega_{0} t-\beta_{m} z\right)\right]
$$

with

$$
\beta_{m} \equiv \beta_{0}+m(2 \pi / L), \quad m= \pm 1, \pm 2, \cdots .
$$

It follows from Eq. (2) that the dispersion characteristics need only be known for $0<\beta_{0}(\omega)<\pi / L$ in order to obtain the dispersion of all the spatial harmonics. A qualitative sketch of the dispersion of a corrugated thinfilm waveguide is shown in Fig. 2. Also shown are points $B$ and $A$ which correspond, respectively, to the $m=0$ and $m=1$ harmonics of a mode whose energy flow is in the $-z$ direction.

A synchronism between an electron beam with velocity $v_{e}$ in the $(+)_{z}$ direction and the $m=1$ spatial harmonic is shown at point $A$. This obtains when

$$
v_{e}=\frac{\omega_{0}}{(2 \pi / L)-\left|\beta_{0}\right|}
$$

The $m=1$ component has oppositely directed phase and group velocity and this, as shown below, can lead to mirrorless tunable optical oscillation.

The coupled mode formalism ${ }^{3-5}$ is a convenient theoretical tool for treating electron-optical beam interactions. The interaction is assumed to take place between the slow space-charge wave of the beam ${ }^{4-6}$ and the $m=1$ spatial harmonic. The latter is represented by a complex amplitude

$$
a_{1} \equiv E_{1} /\left(2 K_{1}\right)^{1 / 2} \beta_{1}
$$

where $E_{1}$ is the electric field of the $m=1$ harmonic at the beam position and the interaction impedance $K_{1}$ is defined by

$$
K_{1}=E_{1} E_{1}^{*} / 2 \beta_{1}^{2} P,
$$

where $P$ is the total power carried by the electromagnetic wave. The slow space-charge mode has a complex amplitude

$$
a_{2}=\left(\omega_{p} v_{e} m / 2 \omega_{0}\left|\rho_{0}\right| e A\right)^{1 / 2} I_{1},
$$

where $\omega_{p}$ is the (reduced) plasma frequency of the beam, $e$ and $m$ are the charge and mass of the electron, $A$ is the cross-sectional area of the beam, and $I_{1}$ the amplitude of the current associated with the slow spacecharge mode. With the above definitions and in the limit of weak coupling, the power in the electromagnetic wave is given by $a_{1} a_{1}^{*}$ while the kinetic power associated with the beam modulation is negative and is given by $-a_{2} a_{2}^{*}$. The coupled mode equations for this case are $e^{4,5}$

$$
\begin{aligned}
& \frac{d a_{1}}{d z}=-i \beta_{1} a_{1}+S a_{2}, \\
& \frac{d a_{2}}{d z}=-i \beta_{2} a_{2}-S a_{1} .
\end{aligned}
$$

The coupling constant $S$ is given by ${ }^{4}$

$$
S^{2}=\beta_{e}^{3} K_{1} I_{0} / 8 \beta_{p} V_{0},
$$

with $\beta_{e} \equiv \omega / v_{e}, \beta_{p} \equiv \omega_{p} / v_{e} . I_{0}$ and $V_{0}$ are, respectively, the beam dc current and acceleration potential. $\beta_{2}$ is the propagation constant of the uncoupled slow space-charge

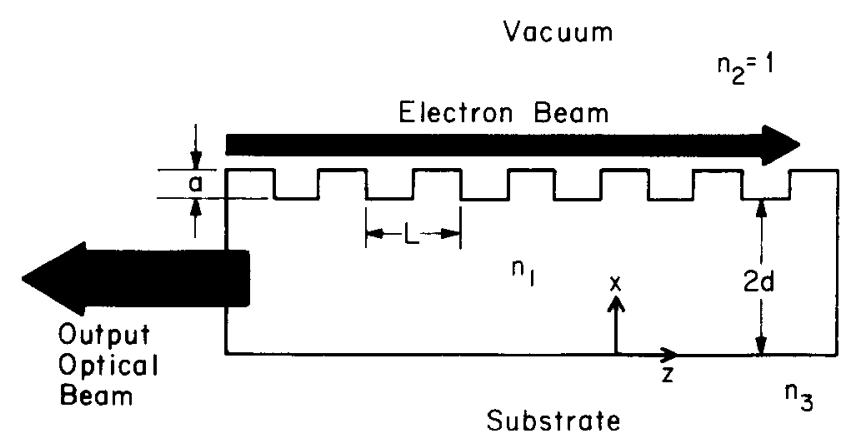

FIG. 1. Schematic diagram of a traveling wave oscillator using a corrugated dielectric thin-film waveguide. 


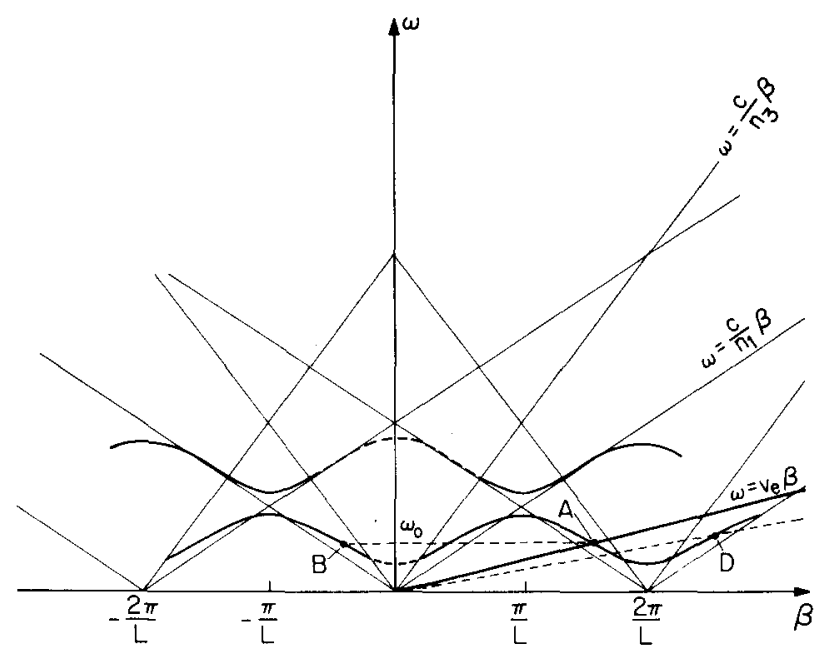

FIG. 2. Schematic representation of the dispersion $(\omega-\beta)$ characteristics of a corrugated thin-film waveguide.

wave and is given by $\beta_{2}=\beta_{e}+\beta_{p}$. We are particularly interested in the possibility of oscillation. Because of the opposite directions of the phase and group velocity of the $m=1$ harmonic, the proper boundary conditions are to specify the field excitation $a_{1}(l)$ at $z=l$ and the beam excitation at $z=0$. We consider the case of an initially unexcited beam and take $a_{2}(0)=0$.

A solution of Eqs. (7) subject to the above boundary conditions yields the following expression for the wave amplification in one traverse:

$$
\begin{aligned}
\frac{a_{1}(0)}{a_{1}(l)}= & \left(-\frac{(\delta-b)}{(\delta+b)} \exp [-i(\delta+b) l]+\exp [-i(\delta-b)]\right)^{-1} \\
& +\left(-\frac{(\delta+b)}{(\delta-b)} \exp [-i(\delta-b) l]+\exp [-i(\delta+b) l]\right)^{-1},
\end{aligned}
$$

where $\delta \equiv \frac{1}{2}\left(\beta_{2}-\beta_{1}\right)$ and $b \equiv\left(\delta^{2}+S^{2}\right)^{1 / 2}$. Of particular interest is the synchronous case $\delta=0$. The solution of Eqs. (7) for this case is

$$
a_{1}(z)=\left[a_{1}(l) \cos (S z) / \cos (S l)\right] \exp \left(-i \beta_{1} z\right),
$$

so that the gain for a section $l$ is

$$
a_{1}(0) / a_{1}(l)=1 / \cos (S l) \text {. }
$$

When $S l=\frac{1}{2} \pi, \frac{3}{2} \pi, \ldots$, the gain becomes infinite so that a finite output is available at $z=0$ with zero input, i.e., oscillation.

An examination of Eq. (9) shows that the oscillation condition can be satisfied only when the mismatch $\delta$ is zero. The oscillation frequency will thus correspond very nearly to the synchronism point $A$ of Fig. 2 so that it can be tuned by varying the electron velocity. The device can also operate as a forward wave amplifier corresponding to synchronism with a forward (i.e. , phase and group velocity in the same direction) spatial harmonic as shown by the inter section point $D$ of Fig. 2. In this case we reverse the sign of $S$ in Eq. (7b) and the solution for $\delta=0$ is

$$
a_{1}(z) / a_{1}(0)=\frac{1}{2}\left(e^{-S_{z}}+e^{s_{z}}\right) \exp \left(-i \beta_{1} z\right) .
$$

An estimate of the gain (or coupling) constant $S$ requires a knowledge of the interaction impedance $K_{1}$ which is the key parameter for this problem. From its definition in Eq. (5) we can write $K_{1}$ as

$$
K_{1}=\left|\frac{E_{1}}{E_{0}}\right|^{2} \frac{\left|E_{0}\right|^{2}}{2 \beta_{0}^{2} P} \frac{\beta_{0}^{2}}{\beta_{1}^{2}},
$$

from which it follows that to calculate $K_{1}$ we need to solve for the field harmonics $E_{m}(x)$. A first-order perturbation solution ${ }^{6}$ of a TM wave propagating in a waveguide such as that shown in Fig. 1 yields

$$
K_{1} \approx \frac{4}{\pi^{2}}\left(\frac{\mu_{0}}{\epsilon_{0}}\right)^{1 / 2} \frac{1}{\left(n_{1}^{2}+1\right)^{4}} \frac{h_{0}^{2} a^{2}}{\gamma \beta_{0}^{2} k w d}\left[\left(\ln n_{1}^{2}\right) \beta_{0}+\frac{1}{2} n_{1}^{2} \gamma_{0}\right]^{2}
$$

where $h_{0}^{2}=n_{1}^{2} k^{2}-\beta_{0}^{2}, \gamma_{0}^{2}=\beta_{0}^{2}-k^{2}, w$ is the width in the $y$ direction.

As an example we consider the case of an oscillator operating at $\lambda_{0}=10 \mu$ using a GaAs thin-film guide $\left(n_{1}\right.$ $\approx 3.5$ ). Taking $v_{e}=\frac{1}{10} c$ we obtain from Eq. (3) a corrugation period of $L \approx 0.75 \mu$. We further assume $w=10 \mu$, $a=1 \mu, d=2 \mu$. The result is

$$
K_{1} \approx 20 \Omega \text {. }
$$

Assuming a total current $I_{0}=0.16 \times 10^{-7} \mathrm{~A},\left(1 \mathrm{~A} / \mathrm{cm}^{2}\right.$ over an aperture of $0.16 \mu \times 10 \mu), V_{0}=3 \times 10^{3} \mathrm{~V}$, we obtain from Eq. (8)

$$
S=22 \mathrm{~cm}^{-1} \text {. }
$$

This implies that for the case of no optical losses the oscillation condition $S l=\frac{1}{2} \pi$ can be satisfied with $l \approx 400 \mu$.

The main practical problem in the way of implementing such oscillators is that of maintaining the close proximity between the beam and the waveguide. The electromagnetic analysis shows ${ }^{6}$ that the field of the space harmonic varies as $\exp \left(-\gamma_{1} x\right)$, where $\gamma_{1}=\left(\beta_{1}^{2}-k^{2}\right)^{1 / 2} \approx 2 \pi / L$, for $\beta_{1} \gg k$. In the case of a slowing-down factor of $\times 10$ as used above we find, using Eq. (3), that the e-folding distance is $\gamma^{-1} \sim \lambda_{0} / 20 \pi$. This suggests that the main potential range of oscillation of this device might be at the longer infrared wavelengths. The feasibility of producing corrugations in thin films with periods down to fractional microns has been recently demonstrated. ${ }^{7}$

The authors acknowledge fruitful discussions with their colleagues, J. R. Pierce, Dr. K. Sakuda, and A. Gover.

\footnotetext{
${ }^{1}$ See, for example, D. A. Watkins, Topics in Electromagnetic Theory (Wiley, New York, 1958).

${ }^{2}$ See, for example, J. R. Pierce, Traveling Wave Tubes (Van Nostrand, Princeton, N. J., 1950).

${ }^{3}$ J. R. Pierce, J. Appl. Phys. 25, 179 (1954)

${ }^{4}$ A. Yariv, Proc. IRE 46, 1956 (1958)

${ }^{5}$ R. W. Gould, IRE Trans. Electron Devices 2, 37 (1955).

${ }^{6}$ A. Gover and A. Yariv (unpublished)

${ }^{7}$ H. L. Garvin, E. Garmire, S. Somekh, H. Stoll, and A. Yariv, Applied Optics (to be published).
} 